\title{
Automatic Transition Predictions Using Simplified Methods
}

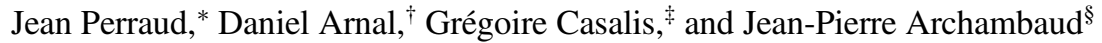 \\ ONERA, 31055 Toulouse, France \\ and \\ Raffaele DonelliI \\ Centro Italiano Ricerche Aerospaziali, 81043 Capua, Italy
}

DOI: $10.2514 / 1.42990$

\begin{abstract}
Laminar-turbulent transition remains a critical issue in a number of cases, including drag reduction, performance prediction of high-lift systems, improved accuracy in general computational fluid dynamics, and reduction of computation cycles for development of optimization tools. Transition delay remains one of the most promising technologies for reducing air transport energy consumption, through natural or hybrid laminar flow control. The use of linear stability theory, either local or nonlocal, remains rather demanding in terms of knowledge and user interaction. Hence, a demand exists for simplified, robust, and accurate transition prediction tools to be inserted into general flow solvers, of boundary-layer or Reynolds-averaged Navier-Stokes types. The problem can be solved by developing transition criteria or database methods. In this last case, characteristics of an actual flow are derived from known solutions of model flows. ONERA, the French Aerospace Laboratory, has long been involved in the development of such methods, and the present paper aims at providing a comprehensive view of the tools developed in the second category, applicable from low-speed two-dimensional to transonic three-dimensional flows, and even to three-dimensional supersonic flows.
\end{abstract}

\begin{tabular}{|c|c|}
\hline & Nomenclature \\
\hline$F$ & $=$ reduced frequency \\
\hline$f$ & $=$ frequency \\
\hline $\mathrm{Hi}$ & $=$ incompressible shape factor \\
\hline$P_{i}$ & $\begin{array}{l}=\text { characteristic parameter for the crossflow } \\
\text { models }\end{array}$ \\
\hline$R_{k}$ & $=$ Reynolds number based on length $k$ \\
\hline & $=$ wall temperature \\
\hline$U e, W e, T e$ & $\begin{array}{l}=\text { velocity components and temperature at the } \\
\text { boundary-layer edge }\end{array}$ \\
\hline$U_{i}$ & $=$ velocity at the location $y_{i}$ of the inflection point \\
\hline$u, w, T$ & $\begin{array}{l}=\text { velocity and temperature components of the } \\
\text { boundary-layer profile }\end{array}$ \\
\hline$V_{g}, \varphi_{g}$ & $=$ modulus and direction of the group velocity \\
\hline$\alpha, \beta$ & $\begin{array}{l}=\text { complex, reduced wave numbers }\left(\alpha=\alpha^{*} \delta_{1}\right) \text {. } \\
\text { Their real parts are the components of the wave } \\
\text { vector } \mathbf{k} .\end{array}$ \\
\hline$\beta^{*}$ & $\begin{aligned}= & \text { crossflow wave number. As } \beta_{i} \text { is usually forced } \\
& \text { to zero, } \beta^{*} \text { represents the real part. }\end{aligned}$ \\
\hline$\delta_{1}$ & displacement thickness \\
\hline$\theta$ & $\begin{aligned}= & \text { angle between the local } x \text { direction and the } \\
& \text { external velocity vector }\end{aligned}$ \\
\hline$\theta_{1}$ & $=$ momentum thickness \\
\hline$\lambda^{*}$ & $\begin{aligned}= & \text { wavelength of the instability } \\
& \lambda^{*}=2 \pi \delta_{1} / \sqrt{\left(\alpha_{r}^{2}+\beta_{r}^{2}\right)}\end{aligned}$ \\
\hline
\end{tabular}

Presented as Paper 1144 at the 47th Aerospace Science Meeting, Orlando, FL, 5-8 January 2009; received 30 December 2008; revision received 22 June 2009; accepted for publication 23 June 2009. Copyright () 2009 by ONERA. Published by the American Institute of Aeronautics and Astronautics, Inc., with permission. Copies of this paper may be made for personal or internal use, on condition that the copier pay the $\$ 10.00$ per-copy fee to the Copyright Clearance Center, Inc., 222 Rosewood Drive, Danvers, MA 01923; include the code 0001-1452/09 and \$10.00 in correspondence with the CCC.

${ }^{*}$ Research Engineer, Models for Aerodynamics and Energetics Department; Jean.Perraud@onera.fr. Member AIAA.

'Director of Research, Models for Aerodynamics and Energetics Department; Daniel.Arnal@onera.fr.

${ }^{\ddagger}$ Professor, Models for Aerodynamics and Energetics Department; Gregoire.Casalis@onera.fr. Member AIAA.

${ }^{\S}$ Research Engineer, Models for Aerodynamics and Energetics Department; Jean-Pierre.Archambaud@onera.fr.

"Research Engineer, Applied Aerodynamics Laboratory, Italian Aerospace Research Center; r.donelli@cira.it.

$\begin{array}{lll}v & = & \text { kinematic viscosity } \\ \rho & = & \text { density } \\ \sigma & = & \text { amplification rate } \\ \varphi & = & \text { angle between the wave vector and the local } \\ & x \text { direction } \\ \varphi_{w} & = & \text { wing sweep angle } \\ \psi & = & \text { angle between the wave vector and the local } \\ & & \text { external velocity vector } \\ \omega_{r} & = & \text { reduced pulsation }\end{array}$

\section{Introduction}

A UTOMATIC and robust laminar-turbulent transition prediction tools are still in demand for improving accuracy of flow computation or development of optimization tools. A number of models have been developed at ONERA, and have been combined into a fairly complete prediction tool which may be inserted into boundary layers or Reynolds-averaged Navier-Stokes (RANS) codes. The aim of the present paper is to provide a comprehensive view of these models, including their use as transition prediction tools inside the 3-D boundary-layer code 3C3D. Selected examples will demonstrate the range of applications by providing comparisons to exact results of linear stability (LST). Ways to implement the database method inside a RANS code will also be discussed, although this remains to be accomplished. Because this paper deals with model presentation and validation, examples will compare $N$ factor curves obtained using the database and exact LST, and will not discuss practical applications involving method calibration, specific data preparation, and statistical analysis of the results. This would grant a separate paper.

The traditional approach for transition prediction is based on the linear stability theory, either local or nonlocal. The addition of the second-order terms curvature and nonparallelism in the nonlocal approach does not significantly improve correlation of computation to experimental results. Therefore, local theory remains widely used for practical applications.

Whereas stability analysis describes how small, preexisting perturbations will grow in the boundary layer through a normal mode response, the $e^{N}$ method [1,2] correlates an amplitude level with the beginning of turbulence. Here, $N=\log \left(A / A_{o}\right)$, where $A / A_{o}$ is the amplitude ratio between the current location and a reference, upstream one. The two most common strategies, envelope and $N_{\mathrm{CF}} / N_{\mathrm{TS}}$, will be discussed later. 
Exact stability calculations are rather demanding in terms of user interaction (no fully automatic code yet) and require a precise description of the boundary layer, imposing conditions on the mesh definition (at least about 40 points in the boundary layer) and on the numerical scheme (low dissipation) in case of a RANS approach. Adequate quality of the boundary-layer profiles may also be ensured using a boundary-layer code. Two separate questions arise: first, the development of simplified stability methods, and second, their coupling to RANS codes.

Concerning the first point, a number of contributions have been made (see [3-12]) that generally fall into the database approach, where solution for an actual boundary-layer profile is obtained from the knowledge of the precomputed stability solutions for a family of profiles, typically of a self-similar family like the Falkner-Skan one in 2-D. The procedure then intends to predict the stability characteristics of actual (nonsimilar) profiles from those of the model profiles. Various procedures and models have been developed that fall into three categories: lookup tables $[\underline{3}, \underline{5}, \underline{12}]$, analytical models $[7,10]$, and neural networks $[8,9]$. In the two first cases, the stability characteristics of a given profile will be determined using an interpolation table or some analytical functions of selected parameters, and in the last case a neural network will provide the desired parameters after a specific training of the network.

The range explored by the family of similar profiles may vary depending on the applications. Because of his interest with interactive boundary layers, Drela [12] introduced in his database 2-D profiles with separation. Other contributors concentrated on attached flows, in relation to direct mode boundary-layer solvers considering configuration without separation. Most published models are restricted to Tollmien-Schlichting (TS) instabilities, with the exception of Fuller et al. [8], Crouch et al. [9], and Langlois et al. [11]. In the first, neural network approach, model parameters include crossflow displacement thickness Reynolds number and a six point sampling of both longitudinal and crossflow velocity profiles. Predicted $N$ factors are within $1 N$-factor count from local stability theory, with the best results in the case of pure crossflow. Langlois uses $w_{\max }$ as the crossflow parameter together with a specific similar solution for 3-D compressible boundary layers. Difficulties apparently stem from the limitation of this family of similar solutions to represent actual profiles computed near an attachment line. In the case of ONERA $[3,4]$, the self-similar Falkner-Skan family was considered for the development of a model for Tollmien-Schlichting instabilities, whereas models for crossflow modes $[7,10]$ were based on typical profiles obtained downstream of the attachment line for a series of low-speed and transonic realistic swept wing flow cases. A similar model was developed for TS instabilities by Stock and Degenhart [5]. In the case of ONERA, three separate models were developed dealing with longitudinal (or TS) instabilities, traveling crossflow (or $\mathrm{CF}$ ) instabilities, and stationary crossflow modes, which we call CF0, respectively.

Concerning the coupling of stability-based methods to RANS codes, several approaches have been explored. ONERA [13] introduced into the elsA code transition criteria for longitudinal and crossflow instabilities. These criteria were developed from results of linear stability and transition correlations. Examples were presented in [14] in the context of high-lift flows. The German Aerospace Center (DLR) [15], the Spanish National Institute for Aerospace Technology (INTA) [16], and others have developed, for wing surfaces, an automatic coupling to a boundary-layer code inside which database methods are used for transition prediction. A presentation of European activities in this field was recently published (see [17]). As there is no example of automatic, internal coupling of a RANS code with a stability approach capable of using directly the RANS velocity profiles, there is still room for much progress.

\section{Linear Stability and the Solution Form}

Consider the swept wing depicted in Fig. 1 , with sweep angle $\varphi_{w}$. A local wing coordinate system is defined taking $x$ as the normal to the leading edge, $y$ the normal to the wall, and $z$ in spanwise direction. The velocity components of the mean flow are $U, W$ in $x$ and $z$

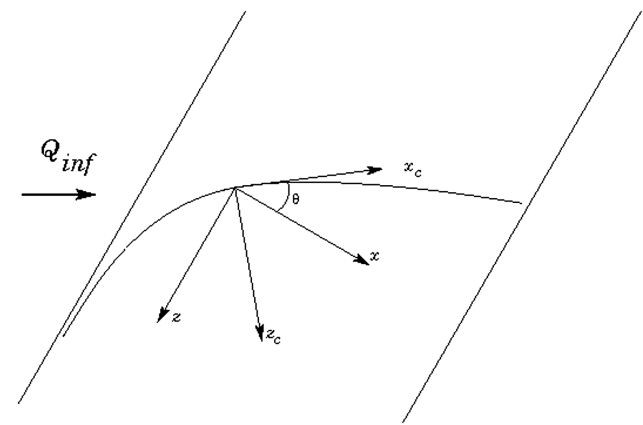

Fig. 1 Wing geometry and coordinates.

directions. The angle between $x$ and $x_{c}$ is $\theta$, with $x_{c}$ the tangent to the external streamline at a point.

In the frame of local stability theory, derivatives $\partial G / \partial x$ and $\partial G / \partial z$ where $G=U, W$ are eliminated from the formulation, that is, forced to zero. The linear growth of small perturbations, added to the base flow, is considered using stability theory. In the context of spatial theory, solutions are written in the form $g(\mathbf{x}, t)=\hat{g}(y) e^{i\left(\alpha x+\beta z-\omega_{r} t\right)}$ where $\omega_{r}=\frac{2 \pi f \delta_{1}}{U_{e}}, \delta_{1}=\int\left(1-\frac{U(y)}{U_{e}}\right) \mathrm{d} y$, and $g$ is any fluctuating quantity.

Real parts of the wave numbers define the wave vector $\mathbf{k}$, see Fig. 2, at angle $\varphi$ from the $x$ direction, and $\psi$ from the local velocity direction: $\varphi=\tan ^{-1}\left(\beta_{r} / \alpha_{r}\right), \varphi=\psi+\theta$. Given these definitions, the LST for a 2-D incompressible flow is the well-known OrrSommerfeld equation, of fourth order, whereas for 3-D compressible flow a sixth-order system is obtained [18]. In 3-D flow, the incompressible equation is

$$
\begin{aligned}
& \left\lfloor v^{\prime \prime \prime \prime}-2\left(\alpha^{2}+\beta^{2}\right) v^{\prime \prime}+\left(\alpha^{2}+\beta^{2}\right)^{2} v\right\rfloor / R_{l}-i\left\lfloor( \alpha U + \beta W - \omega ) \left( v^{\prime \prime}\right.\right. \\
& \left.\left.\quad-\left(\alpha^{2}+\beta^{2}\right) v\right)-\left(\alpha U^{\prime \prime}+\beta W^{\prime \prime}\right) v\right\rfloor=0 \partial
\end{aligned}
$$

with homogeneous boundary conditions on the vertical velocity perturbation $v$ and $v^{\prime}$. Here, the primes denote derivatives with respect to the $y$ coordinate, and $R_{l}=\frac{\rho U_{e} l}{\mu_{e}}$ is a Reynolds number based on a reference length $l$. Because boundary conditions are homogeneous, this is an eigenvalue problem for which solutions only exist for particular combinations of $(\alpha, \beta, \omega)$, referred to as a dispersion relation. Because there are more unknowns than equations, parameters need to be imposed before solving the equations. Typically, ( $\beta$, $\omega)$ or $(\psi, \omega)$ are imposed, and $\alpha$ is obtained as an eigenvalue of the system. The growth rate of solutions is then given by the imaginary part $-\alpha_{i}=\sigma$, and the $N$ factor is obtained by integrating this growth rate.

Several methods exist, referred to as integration strategies. A complete discussion (see $[19,20])$ is outside the scope of this work. In the present context two approaches are considered, the envelope method and the two $N$-factor methods $N_{\mathrm{TS}} / N_{\mathrm{CF}}$, with the following definitions:

The envelope $N$ factor is obtained by integrating the largest amplification rate,

$$
N_{\text {env }}=\max _{f}\left[\int_{x_{o}}^{x} \max _{\psi}(\sigma) \mathrm{d} x\right]
$$

where $x_{o}$ is the critical point in which $\sigma$ becomes positive. The inner maximization may be defined over $\psi, \beta^{*}=\beta_{r} / \delta_{1}$, or $\lambda^{*}$. The

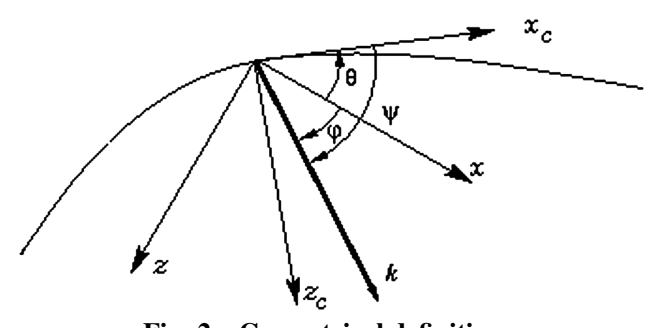

Fig. 2 Geometrical definitions. 
longitudinal $N$-factor is defined by integrating $\left.(\sigma)\right|_{\psi=0}$, considering waves with wave vectors parallel to the outside velocity

$$
N_{\mathrm{TS}}=\max _{f}\left[\left.\int_{x_{o}}^{x}(\sigma)\right|_{\psi=0} \mathrm{~d} x\right]
$$

This definition may be extended to consider longitudinal oblique waves, with

$$
N_{\mathrm{env}}=\max _{f}\left[\int_{x_{o}}^{x} \max _{\psi<\psi_{\max }}(\sigma) \mathrm{d} x\right]
$$

where $\psi_{\max }$ lies in the range $60-80 \mathrm{deg}$. The envelope of envelope crossflow $N$-factor is obtained by evaluating the envelope over various frequencies and either the wavelength or spanwise wave number

$$
N_{\mathrm{CF}}=\max _{f}\left[\max _{\beta^{*} \mathrm{CF}} \int_{x_{o}}^{x}\left(\left.\sigma\right|_{\beta^{*}=c t}\right) \mathrm{d} x\right]
$$

$N_{\mathrm{CF}}$ may be strictly based on stationary modes $(f=0)$, suppressing the outer maximization, or in a concurrent definition may include traveling waves $(f>0) . N_{\text {TS }}$ and $N_{\mathrm{CF}}$ may also be computed based on incompressible stability equations. Whatever the definitions used, each method needs to be calibrated using some experimental database.

As the envelope method was the most commonly used at ONERA, the first database developments were restricted to this approach. An extension to stationary modes and a $N_{\mathrm{TS}} / N_{\mathrm{CF}}$ method were later added with modified definitions for $N_{\mathrm{CF}}$ and $N_{\mathrm{CF} 0}$, as follows:

$$
N_{\mathrm{CF}}=\max _{f}\left[\int_{x_{o}}^{x} \max _{\beta^{*} \mathrm{CF}}(\sigma) \mathrm{d} x\right]
$$

and

$$
N_{\mathrm{CF} 0}=\int_{x_{o}}^{x} \max _{\beta^{*}}\left(\left.\sigma\right|_{f=0}\right) \mathrm{d} x
$$

The maximization is now moved under the integral, corresponding to specialized envelopes. The impact of these definitions is visible in Figs. 3 and 4 , obtained for a low-speed $(80 \mathrm{~m} / \mathrm{s})$ swept $\left(\varphi_{w}=50^{-} \mathrm{deg}\right)$ ONERA-D profile of $0.3 \mathrm{~m}$ chord, at $-1 \mathrm{deg}$ incidence. The database $N_{\text {TS }}$ can be considered equivalent to the exact stability constant $\psi=0 \mathrm{~N}$-factor, and the stationary database $N_{\text {CF0 }}$ can be considered equivalent to the zero-frequency envelope calculation (because only crossflow modes exist at zero frequency), as can be seen in Fig. 3. Figure 4 illustrates the different ways to estimate $N_{\mathrm{CF}}$ and $N_{\mathrm{CF} 0}$ for the same low-speed case. It can be seen that, because of the integration method, the zero-frequency envelope $N$-factor is larger than the $N_{\mathrm{CF}}$ envelope of envelope even though traveling crossflow modes are more unstable than stationary ones. Very different results are obtained depending on the definition; hence, for transition prediction, calibration must be determined for each formulation.

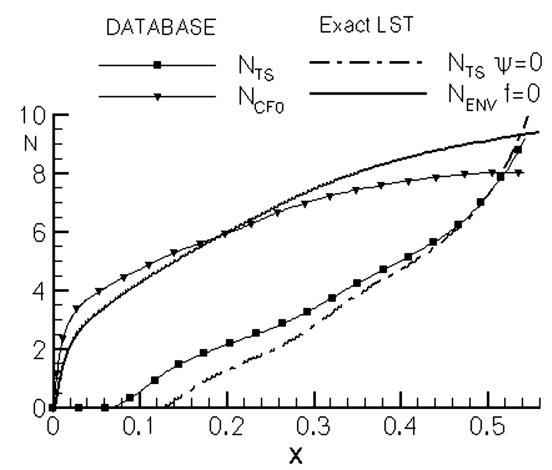

Fig. 3 Comparing $N_{\text {TS }}$ and $N_{\text {CF0 }}$ for LST and database for a low-speed case.

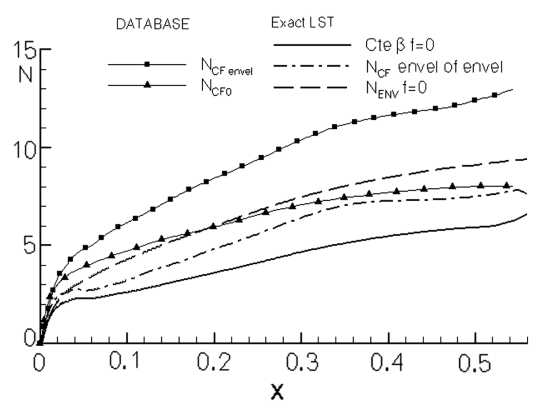

Fig. 4 Consequences of various definitions for $N_{\mathrm{CF}}$.

\section{Database Approach for Longitudinal Instabilities}

Longitudinal, or TS instabilities, are governed by viscosity. A first model of such instabilities for 2-D low-speed flow was proposed by Vialle and Arnal [4] in 1984 based on a set of exact stability solutions of attached Falkner-Skan self-similar profiles in 2-D flow. The incompressible shape factor $H i=\delta_{1} / \theta_{1}$, where $\theta_{1}=\int \frac{U(y)}{U_{e}}(1-$ $\left.\frac{U(y)}{U_{e}}\right) \mathrm{d} y$ is the momentum thickness, was used as the key parameter.

The simplified stability approach, or database method, provides an estimation of the growth rate $\sigma$ directly from mean flow parameters and the boundary-layer profile characteristics. The starting idea is that the Reynolds number variation of growth rates obtained solving the exact Orr-Sommerfeld equations can be represented, for a given profile, using two half parabolas as shown in Fig. 5. Extension to decelerated flow $(H i>2.59)$ can be obtained using an added inviscid parabola, as shown in Fig. 6 . The effect of compressibility was introduced into the model, using the external Mach number as an additional parameter [3].

For a given mean flow $\left(H i, M_{e}\right)$ and reduced frequency $F=\frac{2 \pi f \mu_{e}}{\rho_{e} U_{e}^{2}}=\frac{\omega_{r}}{R_{\delta_{1}}}$, the amplification curve is approximated as

$$
\begin{gathered}
\sigma=\max \left[\sigma_{V}, \sigma_{I}\right] \quad \sigma_{V, I}=\sigma_{M}\left(1-\left[\frac{R_{\delta_{1}}-R_{M}}{R_{K}-R_{M}}\right]^{2}\right) \\
R_{K}=\begin{array}{ll}
R_{0} & \text { if } R_{\delta_{1}}<R_{M} \\
R_{1} & \text { if } R_{\delta_{1}}>R_{M}
\end{array}
\end{gathered}
$$

where $\sigma_{M}, R_{0}, R_{1}$, and $R_{M}$ are associated with both the viscous and the inviscid parabolas of Fig. $\underline{6}$.

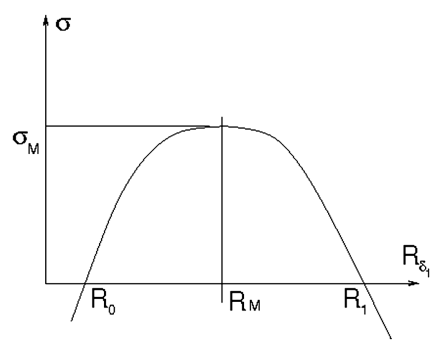

Fig. 5 Basic parabola model.

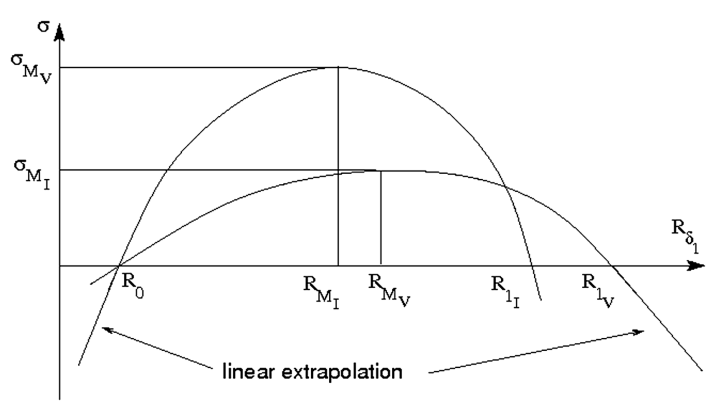

Fig. 6 Extended parabola model for TS waves. 


$$
\begin{aligned}
& R_{M}=k_{1} F^{E_{1}} \quad \text { for viscous or inviscid parts } \\
& R_{1}=k_{2} F^{E_{2}} \quad \sigma_{M_{I}}=B_{I}\left(1-\frac{F}{F_{o I}}\right) \\
& R_{0_{V}}=R_{0_{I}}=R_{M_{V}}\left(1+C\left(F_{c}-F\right)\right) \quad \\
& \sigma_{M_{V}}=\min \left\{B_{V}\left(1-\frac{F}{F_{o_{V}}}\right), \sigma_{M_{V_{\mathrm{MAX}}}}\right\}
\end{aligned}
$$

Using these definitions, 15 parameters need to be determined as functions of $\mathrm{Hi}$ and $\mathrm{Me}$ :

$$
\left(B, F_{o}, K_{1}, E_{1}, K_{2}, E_{2}\right)_{I, V}, \quad C, \quad F_{c}, \quad \sigma_{M_{V_{\mathrm{MAX}}}}
$$

This is realized using a two-entry lookup table. Amplification rates are then obtained as a function of the Reynolds number $R_{\delta 1}$ based on the incompressible (respectively compressible) displacement thickness for incompressible (respectively compressible) flow. Moreover, the effect of wall temperature $T_{w}$ can be modeled using four more parameters modifying the previous 15 .

Tables are defined for $2.22<H i<4.023$ and $0<M e<1.3$. Use is limited to profiles without backflow. To calculate stabilizing regions, where $N$-factors decrease, extension to stable amplification rates has been added by extrapolating the parabolas with a line tangent at the zero crossing point, as shown in Fig. $\underline{6}$.

There are two ways to use this model. First, as originally conceived, it can be applied to the velocity component profile in the direction of the external streamline. This defines a TS amplification rate, which can be integrated into $N$-factor curves, producing the $N_{\text {TS }}$ $N$-factors after a frequency envelope. For transonic flows, the amplification rates generated by the method correspond to the most unstable oblique wave in exact calculation.

Second, velocity profiles projected in the $\Psi$ direction can be considered, using Gaster's relation and the Stuart theorem in the way described in the next section. This allows optimization in the $\Psi$ direction, and can be used to define an envelope $N$-factor (still limited to viscous instabilities). This second method slightly improves the longitudinal $N$-factor computation, and generates a $\Psi$ dependence. It may also complement the computation of the envelope by including highly oblique longitudinal waves (see Fig. 7 and corresponding discussion).

\section{Simplified Model for Inflectional Instabilities}

Crossflow instabilities are inflectional in nature, and they are determined by the location and characteristics of the velocity profile inflection point. Considering Falkner-Skan 2-D profiles with reverse

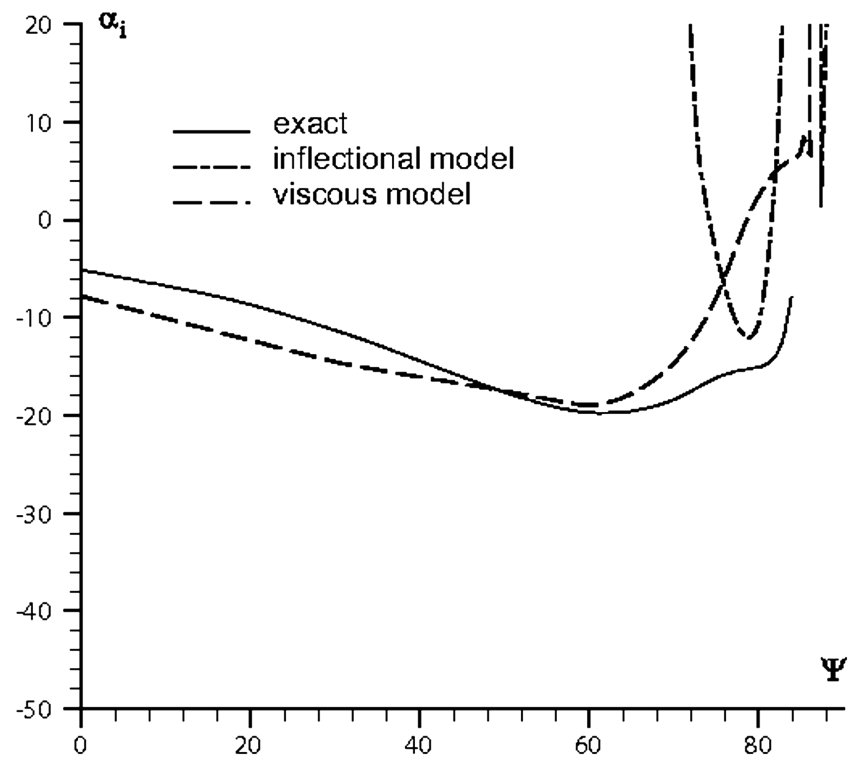

Fig. 7 Comparing viscous and inflectional models to the exact amplification (3-D low-speed case).

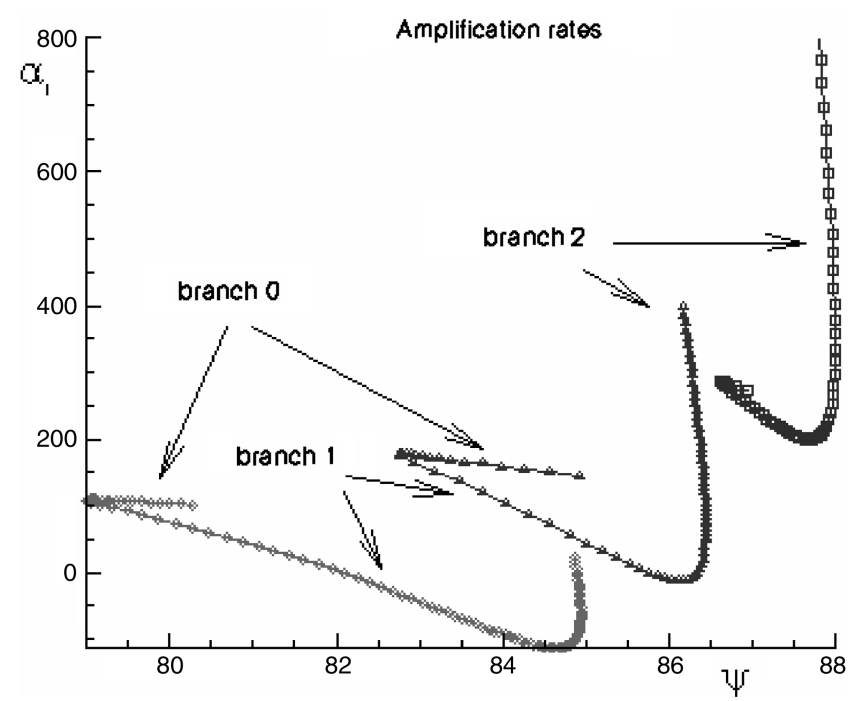

Fig. 8 Zero-frequency exact stability solutions for three stations.

flow near the wall, it was first shown [21] in 1986 that two parameters had to be used, related to the characteristics of the inflection point. These were $U_{i}=u\left(y_{i}\right)$ and $P_{i}=y_{i}\left(\frac{\partial u}{\partial y}\right)_{y_{i}}$, where $y_{i}$ is the location of the highest inflection point. A first model was developed based on similar profiles that was found inadequate when looking at inflectional profiles like those causing crossflow instabilities in 3-D boundary layers. A new model was then created during the ELFIN II project by Casalis and Arnal [7] based on a series of actual profiles computed near the attachment line of swept profiles, and successfully applied to propagating crossflow instabilities.

To extend a 2-D model to 3-D mean flow, use of Stuart's theorem [22] and Gaster's relation [23] are necessary. In temporal theory, and for incompressible flow, Stuart's theorem states that the growth rate $\sigma_{\phi}$ in any direction $\phi$ can be determined from the stability of the 2-D velocity profile resulting from the projection of the original 3-D velocity in that same $\phi$ direction. This projected 2-D profile is defined as $U_{\phi}=\frac{\alpha_{r}}{k} U+\frac{\beta_{r}}{k} W$, where $k=\sqrt{\alpha_{r}^{2}+\beta_{r}^{2}}$ is the modulus of the wave vector $\mathbf{k}$. This applies in the case of temporal theory, whereas boundary-layer stability usually uses spatial theory. Gaster's relation gives a relation expressing spatial growth rate in some $\phi$ direction in terms of temporal amplification $\omega_{i}$, group velocity modulus $V_{g}$, and direction $\varphi_{g}$. The group velocity direction is $\varphi_{g}$ with respect to the local $x$ direction. In most cases, $\varphi_{g}$ remains very close to $\theta$, within a few degrees (i.e., the group velocity direction remains very close to that of the external velocity). This property is used here, as well as in many instances of exact LST resolutions. Gaster's relation may be written as

$$
-\sigma_{\varphi}=\frac{\omega_{i}}{V_{g} \cos \left(\varphi-\varphi_{g}\right)}
$$

In $x$ direction, $\varphi=0$ and $-\sigma_{x}=\frac{\omega_{i}}{V_{g} \cos \left(\varphi_{g}\right)}$.

The amplification $-\sigma_{x}$ may be expressed in terms of $-\sigma_{\varphi}$ computed in some $\varphi$ direction

$$
\sigma_{x}=\sigma_{\varphi} \frac{\cos \left(\psi+\theta-\varphi_{g}\right)}{\cos \left(\varphi_{g}\right)}
$$

and, noting $\varphi_{g} \approx \theta$,

$$
\sigma_{x} \approx \sigma_{\varphi} \frac{\cos (\psi)}{\cos (\theta)}
$$

This relation gives the amplification in $x$ direction, used for $N$-factor integration, as a function of the amplification in the $\varphi$ direction, computed using the velocity profile projected in that direction.

In the case of an inflectional profile, the amplification rate is defined as before 


$$
\sigma=\sigma_{M}\left(1-\left[\frac{R_{\delta_{1}}-R_{M}}{R_{j}-R_{M}}\right]^{2}\right) \quad R_{j}=\begin{array}{ll}
R_{0} & \text { if } R_{\delta_{1}}<R_{M} \\
R_{1} & \text { if } R_{\delta_{1}}>R_{M}
\end{array}
$$

now with the following definitions:

$$
\begin{array}{ll}
\sigma_{M}=a F+b \sqrt{F}+\sigma_{0} & R_{M}=k_{M} F^{-m_{M}} \quad R_{0}=k_{0} F^{-m_{0}} \\
R_{1}=k_{1} F^{-m_{1}} &
\end{array}
$$

where $a, b$, and $\sigma_{0}$ are analytical functions of $U_{i}, P_{i}$, and the $(k, m)$ coefficients are functions of $\sigma_{0}$.

Extension to compressible flow has been achieved by using the generalized inflection point, $\frac{\partial}{\partial y}\left(\rho \frac{\partial u}{\partial y}\right)=0$, and introducing the density value into $P_{i}: P_{i}=\rho\left(y_{i}\right) y_{i}\left(\frac{\partial u}{\partial y}\right)_{y_{i}}$.

In the case of multiple inflection points, the highest is always selected. In regions where modes are damped, growth rates are again approximated using linear extensions of parabolas, as shown in Fig. 6. The final model applies only to traveling crossflow instabilities $(F>0)$, and in a range $|\psi|<\psi_{\max }<90 \mathrm{deg}$. In practice, $\psi_{\max }$ is set between 88.5 and $89 \mathrm{deg}$. For nonzero frequencies, the two models for longitudinal and crossflow instabilities were later combined. The resulting database method produces, with high efficiency, an estimation of the stability characteristics of 3-D boundary layers. Figure 7 compares the TS and CF growth rates to exact LST solutions, for a swept wing low-speed case at a given nonzero frequency. The method of profile projection is used here for both TS and CF. It can be seen in Fig. 7 that database TS growth rates remain close to exact growth rates up to about $\psi=70 \mathrm{deg}$, and misses completely the CF contribution for larger $\psi$. On the other hand, around $\psi=80 \mathrm{deg}$, the CF model produces a good estimation of the most amplified CF instability. In general, when unstable modes exist the TS models produce quite a good approximation of $\sigma(\psi)$ over a wide $\psi$ range, whereas the CF model only produces a good estimation of $\sigma_{\mathrm{CF}, \max }$.

\section{Stationary Modes}

The previously described model is strictly limited to traveling instabilities, and indeed its precision increases with frequency. It is well-suited for transition predictions on transonic swept wings, but does not allow comparisons to results obtained using two $\mathrm{N}$-factor methods. With the objectives of extending the previous model, and to allow such comparison, stationary modes were later considered.

This model was created based on exact stationary stability solutions of a set of low-speed and transonic wing flows, with validation using quite an extensive set of cases. This part was realized

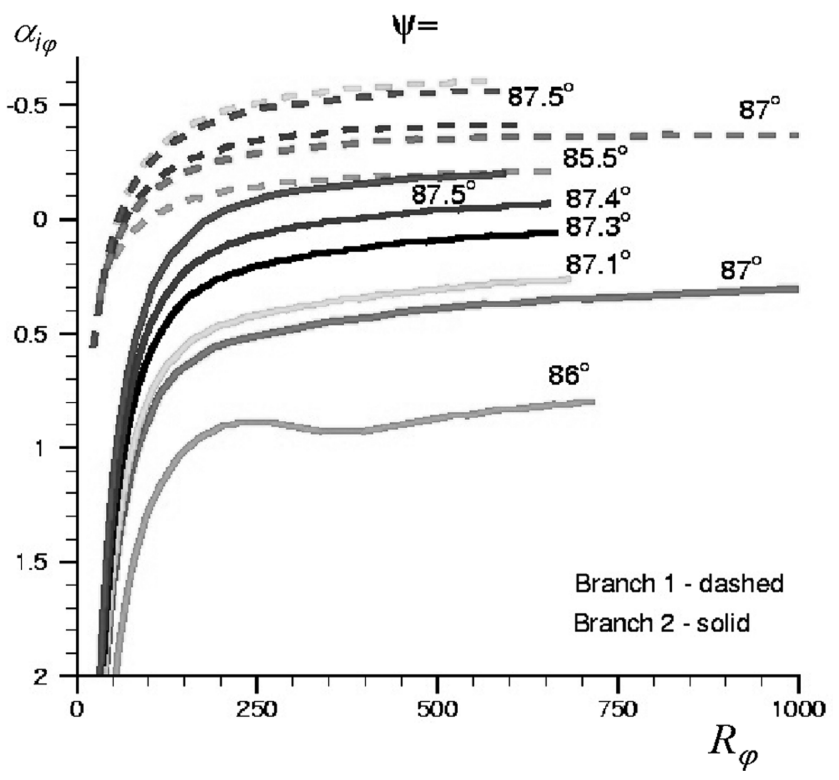

Fig. 9 Reynolds variations of the two branches. by Perraud and Donelli [10] in the application of hybrid laminar flow technology on transport aircraft (ALTTA) European project. In this case, the maximum growth rate is generally located at the junction of branches B1 and B2, as shown in Fig. $\underline{8}$, for exact stationary solutions for three stations of a low-speed case $\left(60^{\circ}\right.$ swept ONERA-D profile). At each station, the solution curve is obtained by varying $\beta^{*}$ from a small value up to about $8000 \mathrm{~m}^{-1}$. In the selected representation $(\psi$, $\alpha_{i}$ ), these solutions are composed of three branches: B0 corresponding to small $\beta^{*}$, then $\mathrm{B} 1$ and $\mathrm{B} 2$ for increasing $\beta^{*}$. The largest amplification is observed at the junction of B1 and B2 (between 82 and $85 \mathrm{deg}$ at the first station), hence only these two branches need to be considered in a model.

In this case, the Reynolds variations of projected amplification rates should not be represented by parabolas, but by hyperbolas as visible in Fig. 9. Plotting the variations of $\alpha_{i} R_{\varphi}$ versus $R_{\varphi}$ produces almost straight lines, hence amplifications can be estimated as $\alpha_{i \varphi} R_{\varphi}=A . R_{\varphi}+B$.

Based on the results shown in Fig. $\underline{9}$, this expression may be written for branch one as $\alpha_{i \varphi}=\sigma_{\infty}\left(1-R_{c} / R_{\varphi}\right)$, where $\sigma_{\infty}$ represents the asymptotic value of the growth rate when the Reynolds number $R_{\varphi}$ tends to infinity, and $R_{c}$ is a critical projected Reynolds number. These two parameters are equivalent to the coefficients $A$ and $B$ in the previous equation. Parametric variations show that $R_{c}$ essentially depends on parameter $P_{i}$, whereas $\sigma_{\infty}$ is a function of $U_{i}$.

The two branches need to be modeled independently. In both cases, $\sigma_{\infty}$ is represented using a polynomial expression function only of $U_{i}$, of third order for branch one and second order for branch two. An exponential function of $P_{i}$ is used for $R_{c}$ for branch one, and again a second-order polynomial in $R_{c}$ for branch two.

An additional condition, based on $P_{i}$, allows to determine an upper $\psi$ limit. A spline function is used to represent the junction between the two branches, because using the crossing point between the two branches would strongly overestimate the growth rate. The local extremum gives the maximum growth rate and the corresponding $\psi$. This produces the largest stationary growth rate at any given station, so that the $N_{\mathrm{CF}}$ is equivalent to an envelope over all values of the crossflow wave number $\beta^{*}$.

Figure 10 shows a comparison of the exact and modeled amplification rates for a typical transonic case for three different stations. Actual amplification rates (for the 3-D profile) are shown, for which a very good agreement is obtained.

\section{Methods for Transition Prediction}

Shooting methods are the classical tools for solving this type of problem, but they require the initial guess of a solution that should not be too far from the real solution. This initial guess creates a

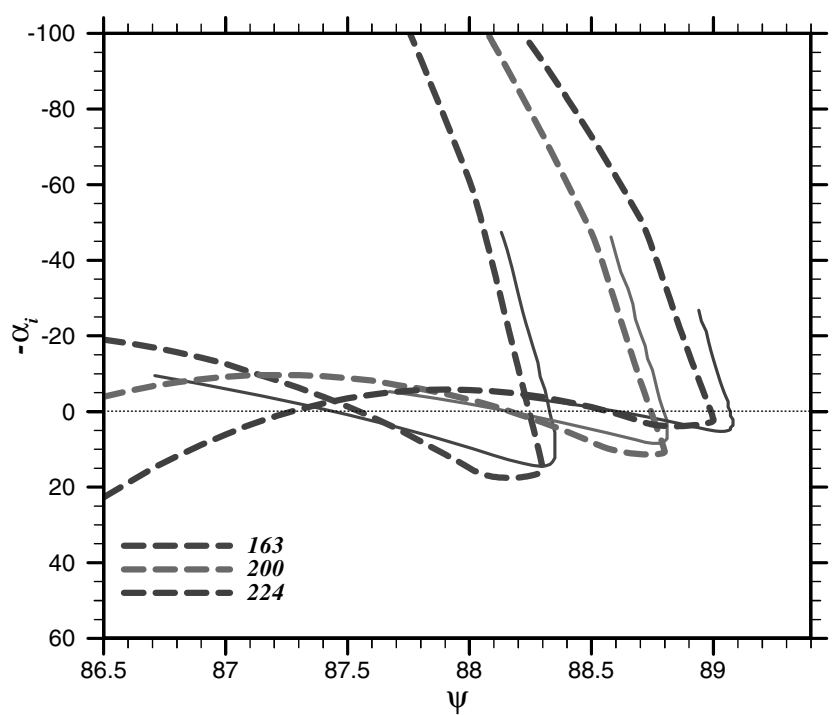

Fig. 10 Exact and modeled growth rate curves (thin line represents exact, dashed line represents model). 


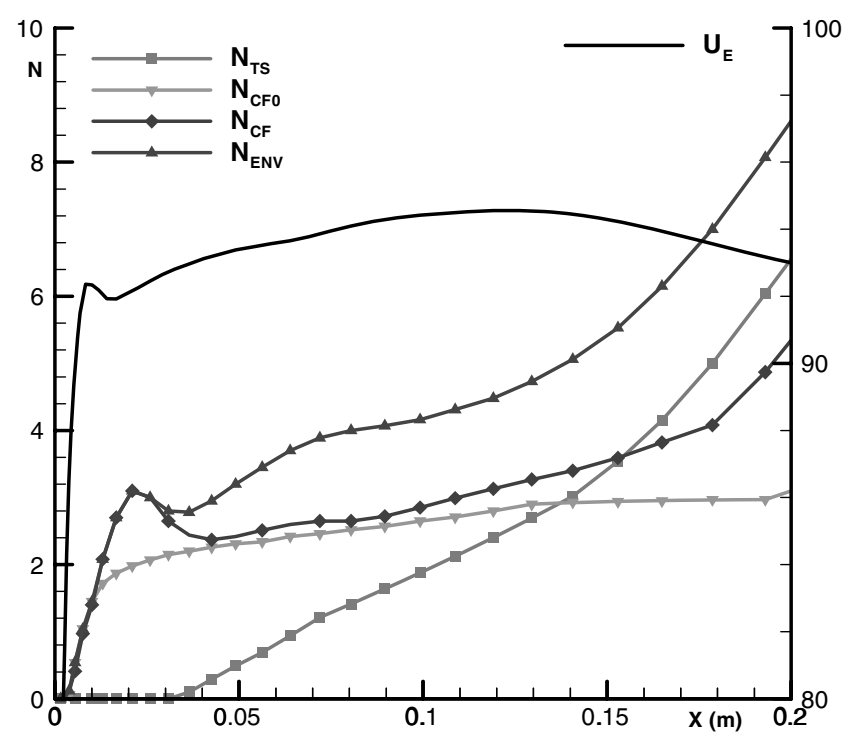

Fig. 11 Application to a low-speed swept wing: database generated $N$ factors and velocity distribution.

difficulty in applying these methods. The TS growth rates only depend on integral boundary-layer parameters and Reynolds and Mach number, and the CF and CF0 mostly depend on the characteristics of the highest inflection point in the velocity profile. The three models were then combined to produce growth rates for the various kinds of boundary-layer instabilities. These growth rates may be integrated to produce envelope $N$-factors exactly as in the case of exact stability theory,

$$
N_{\mathrm{env}}=\max _{f}\left(\int \max \left(\sigma_{\mathrm{TS}}, \sigma_{\mathrm{CF} 0}, \sigma_{\mathrm{CF}}\right) \mathrm{d} x\right)
$$

In practice, a method based on the Fibonacci series is used to locate the wave direction producing the largest growth rate after the definition of the range of variation of the $\psi$ angle. This is done separately for $N_{\mathrm{TS}}$ and $N_{\mathrm{CF}}$. In the case of the envelope, careful optimization has ensured that, in general, the most amplified frequencies are represented with a precision of about one count in $\mathrm{N}$ factor, for $N \approx 10$, or about $10 \%$. The transition $N$-factor must then be determined exactly as it is determined when using an exact stability code, with values at transition depending on the type of environment (flight or wind-tunnel) and of the dominant type of instability, see [20].

The two $N$-factors' transition prediction method may also be used, with two options. The first tries to resemble the classical $N_{\mathrm{TS}} / N_{\mathrm{CF}}$ method, based on $N_{\mathrm{CF} 0}$ and $N_{\mathrm{TS}}$ with $\psi=0$ deg (but uses the compressible approach for compressible flows). The second takes into account a larger set of modes, as it includes traveling waves into $N_{\mathrm{CF}}$.

Concerning crossflow models, there remains a lack of precision for low frequencies at the junction of the two models. Growths of lowfrequency traveling crossflow instabilities are underestimated, but zero-frequency modes are correctly estimated. This does not seriously impact the $N$-factor curves and the quality of transition prediction.

The resulting code allows complete stability calculation with about 2 orders of magnitude reduction in computing time compared to exact stability calculation. Another important advantage of the method is that it does not require any initial values, and is thus well adapted for insertion into boundary-layer codes for fully automatic usage, like it has been done in the ONERA 3-D boundary-layer code 3C3D, but also in codes used by the European Research Centers Instituto Nacional de Tecnica Aeroespacial (INTA, Spain), Swedish Defense Research Agency (FOI, Sweden), Centro Italiano Ricerche Aerospaziali (CIRA, Italy), and DLR (Germany), in the course of the European projects EUROLIFT, EUROLIFT 2, and SUPERTRAC. In the case of $3 \mathrm{C} 3 \mathrm{D}$, this allows the stability computation over the complete surface of a vehicle, with a 3-D mean flow (local stability hypotheses are still contained in the models).

Regarding the coupling to RANS codes, several difficulties have to be addressed: first, assessing the conditions to obtain an adequate precision of the velocity profiles and boundary-layer description; second, reducing the computation time to become compatible with iterative formulations; and third, allowing the transition to move in both upstream and downstream directions in the course of the computation. A number of tests have been conducted regarding the first point; required precision may be obtained using a Roe scheme with low-dissipation settings, whereas a centered Jameson scheme is not adapted, even with very small numerical dissipation. The number of points in the boundary layer should be at least about 40, avoiding too much wall clustering. The second and third points have been treated already with the use of transition criterion and the automatic coupling to a boundary-layer code for swept wing flows. It should be noted that although the TS model could be adapted to the RANS precision without much difficulty, the two crossflow models definitely require a precise description of the inflection point.

\section{Applications}

The first example corresponds to a low-speed $(80 \mathrm{~m} / \mathrm{s})$ swept $\left(\varphi_{w}=50 \mathrm{deg}\right)$ ONERA-D profile of $0.3 \mathrm{~m}$ chord, at $-1 \mathrm{deg}$ incidence (already used for Figs. 3 and $\underline{4}$ ). The velocity distribution in Fig. 11 shows a small peak on the upper side near the leading edge, followed by a negative pressure gradient. For the sake of clarity, only database results are shown in Fig. 11 for this case. Stationary and traveling crossflow instabilities are observed near the leading edge, in the negative pressure gradient region. Quite typical for this kind of flow, stationary instabilities dominate crossflow along 5-7\% of chord, whereas traveling waves become larger further downstream. TS instabilities ( $\psi=0 \mathrm{deg})$ start to grow at about $15 \%$ chord, where the negative pressure gradient becomes less pronounced and rise more rapidly past the maximum velocity point. The four instability curves correspond to $N_{\mathrm{TS}}, N_{\mathrm{CF} 0}, N_{\mathrm{CF}}$, and $N_{\mathrm{ENV}}$. Concerning the envelope $N$-factor, crossflow contributes to its first part, roughly up to the maximum velocity point, and then TS waves are the dominant contribution. All the curves are envelope overfrequency except for the stationary crossflow.

It should be kept in mind that this $N_{\mathrm{CF} 0}$ is comparable to a zerofrequency envelope $N$-factor, by definition with larger values than what would be obtained with LST based on constant $\beta^{*}$ method (cf. Fig. 4).

It is quite obvious from Fig. 11 that transition will correlate to different $N$-factor values depending on the definition. Although a

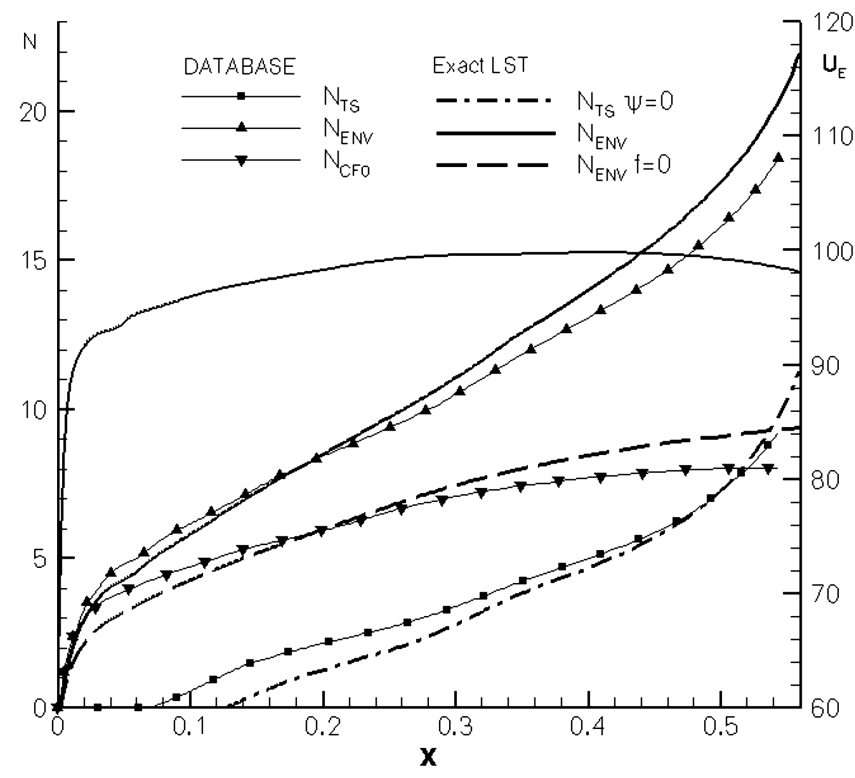

Fig. 12 Application to a low-speed case: comparison to exact LST. 
single $N$-factor is used with the envelope method, a curve has to be defined in the case of two $N$-factor methods, transition being predicted when the point of coordinates $\left(N_{\mathrm{CF}}, N_{\mathrm{TS}}\right)$ crosses it. See [24] for examples of application.

A second case, corresponding to a low-speed DTP-B wing model at $40 \mathrm{deg}$ sweep is next considered $\left(U_{\infty}=70 \mathrm{~m} / \mathrm{s}, \alpha=-4 \mathrm{deg}\right)$. Figure 12 shows the velocity distribution and compares database $N$ factors with their exact LST counterparts. Again, this upper side at negative incidence shows an extended negative pressure gradient without any leading-edge peak, which promotes crossflow instabilities. TS waves start further downstream, resulting in a mixed CF and TS case. Comparison to exact LST results shows differences below two points in $N$-factors, or about $10 \%$, on the envelope curve, and even smaller for $N_{\mathrm{TS}}$ and $N_{\mathrm{CF}}$.

A third example, presented in Fig. 13 with the same curves as Fig. 12, corresponds to a transonic case from the Fokker 100 natural laminar flow flight experiments, which were run in the frame of the European project ELFIN [19]. Boundary layers were computed using a conical wing hypothesis, whereas stability calculations were obtained here assuming an infinite swept wing. The vertical arrow indicates where transition occurs, and the velocity distribution is also plotted on the figure. The database results are again compared with exact LST computations, with a fair agreement in this difficult test case. Departure from exact results remains lower than two at the transition location, but with increasing difference as the database $N_{\mathrm{CF} 0}$ remains at a constant value while the LST result continues growing.

Five similar Fokker 100 cases were analyzed, bringing out the following correlations at transition in flight conditions: $N_{\mathrm{TS}}=9$, $N_{\mathrm{CF}}=15, N_{\mathrm{CF} 0}=11$, and $N_{\mathrm{env}}=22$ for TS-dominated cases, and $N_{\text {env }}=15$ for crossflow-dominated cases.

In wind-tunnel studies with the proposed database method, typical values for crossflow cases are $N_{\mathrm{CF}}=7$ and $N_{\mathrm{CF} 0}=5$, but these values should be adapted for each test.

Application to transonic cryogenic tests (transonic small models at large Reynolds numbers) showed that the crossflow model is not well-adapted at present because boundary layers become extremely thin, outside the validity domain of the model. On the other hand, the $N_{\mathrm{TS}} / N_{\mathrm{CF} 0}$ approach remains valid in these configurations.

After application to a number of swept profiles, the complete database method has been introduced as a transition prediction tool into the 3C3D boundary-layer code [25], allowing the computation of amplification for 3-D flows over the complete surface of a wing or a vehicle. In 3C3D, growth rates are calculated in the course of the parabolic boundary-layer equations resolution, and then $N$-factors are integrated along external streamline directions, until transition (or a separation) is predicted. Note that $3 \mathrm{C} 3 \mathrm{D}$ also includes a transition zone calculation based on an intermittency function. To illustrate the use of this tool, an application to the slat of a high-lift configuration is presented in Fig. 14. The KH3Y configuration was extensively studied within the European projects EUROLIFT I and II [14]. Results are presented here for the upper slat, for a wing incidence of $8.5 \mathrm{deg}$ and a chord Reynolds number of $4.15 \times 10^{6}$.

Three methods are used in this case, envelope, $N_{\mathrm{TS}} / N_{\mathrm{CF}}$, and $N_{\mathrm{TS}} / N_{\mathrm{CF} 0}$. In the first case, the three models (for TS, CF, and CF0) contribute to the envelope curve. In the second case, $N_{\mathrm{CF}}$ includes waves with a $\psi$ direction such that $|\psi|<60^{\circ}$, and $N_{\mathrm{CF}}$ is based on traveling and stationary waves. In the last one, $N_{\mathrm{TS}}$ is restricted to $\psi=0^{\circ}$ and $N_{\mathrm{CF} 0}$ to $f=0$. Figure 14 shows the various regions of the flow according to the three methods, but with the $N$-factors at transition given in Table 1 .

The outer slat is characterized by a large region (25\% of span) where transition is caused by laminar separation. On each side of this separated zone, transition is predicted by $3 \mathrm{C} 3 \mathrm{D}$ between 30 and $50 \%$ chord, followed by an extended transition region up to the trailing edge. Early transition is only predicted near the slat root with $N_{\mathrm{TS}} / N_{\mathrm{CF} 0}$, resulting in a transition zone followed by a turbulent one. In each case, the unstable region is delimited into TS and CF regions, depending on the largest of the two $\mathrm{N}$-factors. Comparison of the results seems to show the equivalence of the three methods provided that proper settings are used for transition $N$-factors. It would be most

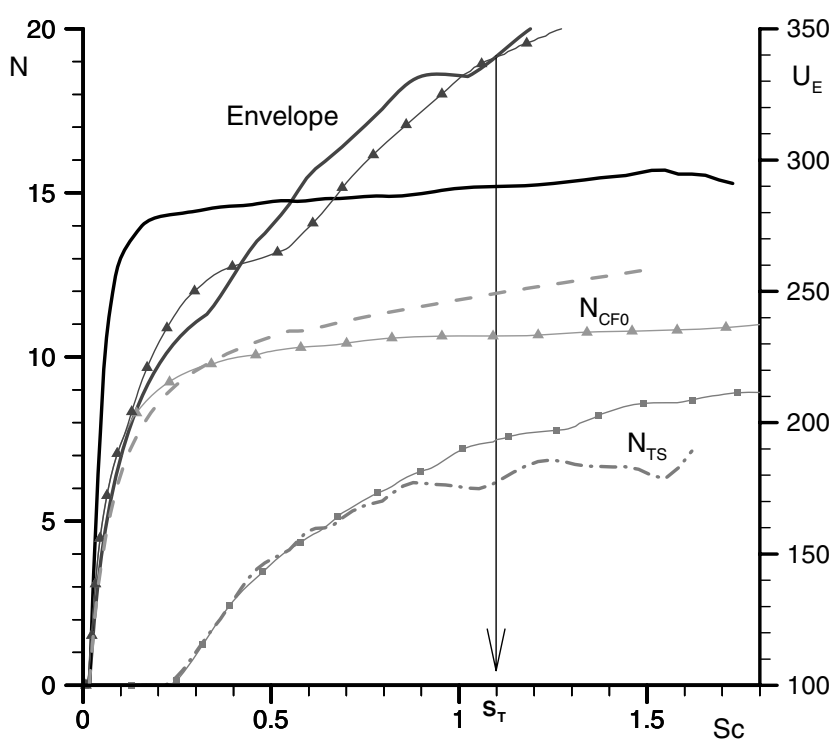

Fig. 13 Application to a transonic Fokker 100 flight-test case. (Same legend as Fig. 12. Arrow shows the transition location).

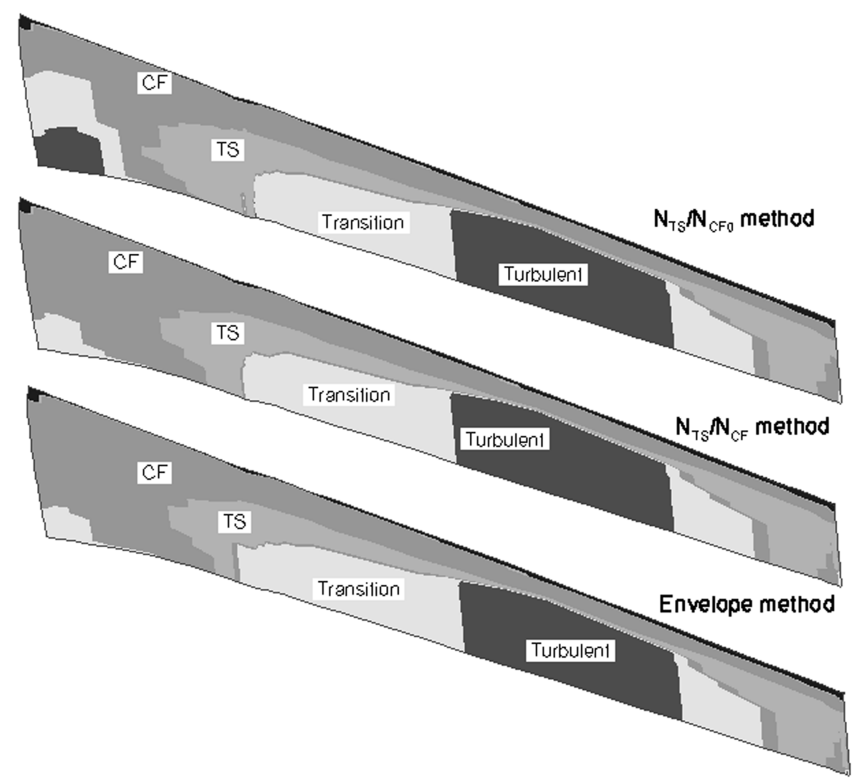

Fig. 14 Application to the upper side of the KH3Y slat $\left(8.5^{\circ}\right.$ incidence, $R e_{C}=4.1510^{6}$.

interesting to compare the spanwise evolution of transition with experimental results, but in most experiments transition measurements are usually done in a limited spanwise region.

A final example is presented in Fig. 15 that corresponds to a Mach 2 highly swept $\left(\varphi_{w} \approx 60 \mathrm{deg}\right)$ laminar wing designed and flight tested by the Japan Aerospace Exploration Agency (JAXA) [26]. ONERA cooperated with JAXA on the pre- and postflight numerical transition analysis and performed wind-tunnel tests in the ONERA S2. Figure 15 shows, for a postflight case, a comparison of envelope $N$-factor curves obtained with two exact stability codes and with the database method. The agreement on the envelope curves is here again very satisfying. An extended version of the TS model was

Table 1 Transition $N$-factor settings for the KH3Y slat

\begin{tabular}{ccc}
\hline \hline & $N_{\mathrm{ENV}}$ or $N_{\mathrm{TS}}$ & $N_{\mathrm{CF}}$ \\
\hline Envelope & 7.15 & \\
$N_{\mathrm{TS}} / N_{\mathrm{CF}}$ & 7.15 & 7 \\
$N_{\mathrm{TS}} / N_{\mathrm{CF} 0}$ & 7.15 & 5 \\
\hline \hline
\end{tabular}




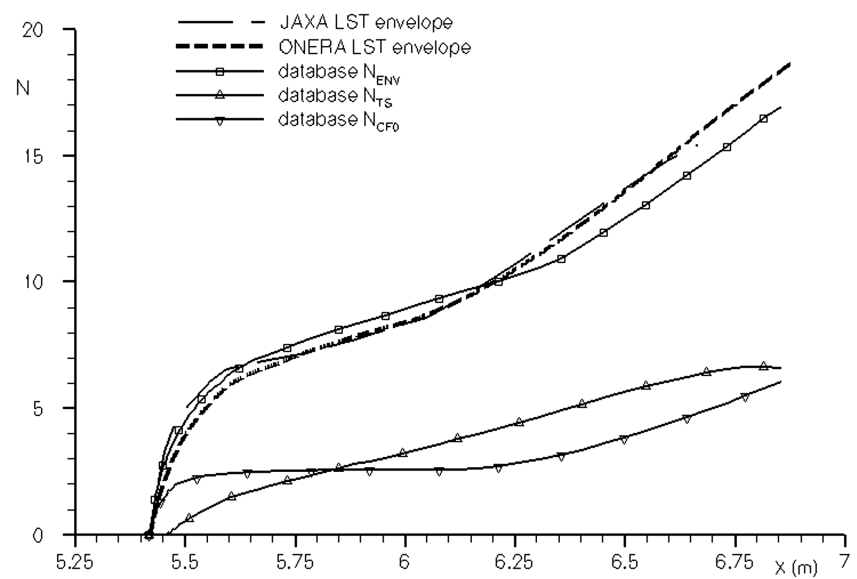

Fig. 15 Application to the Mach 2 JAXA wing; comparison to exact LST.

used for this case, which includes the wall temperature $T_{w}$ as an additional parameter. $N_{\mathrm{TS}} / N_{\mathrm{CF}}$ curves are also shown but not compared to exact calculation. Nevertheless, these curves confirm that crossflow is effectively contributing to the most upstream instabilities, and is quite correctly predicted even for this supersonic case.

\section{Conclusions}

Three separate models were developed for simplified prediction of longitudinal (TS), traveling crossflow (CF), and stationary instabilities (CF0), aimed at allowing fast and automatic transition predictions. These three models rely heavily on Stuart theorem, stating in temporal theory the equivalence of the stability characteristics of a 3-D velocity profile in direction $\psi$ with that of the projected 2-D profile in the same direction, and on Gaster's relation to transform the spatial growth rates. Although integration should in principle be conducted along the group velocity direction, it is assumed that this direction remains very close to the external velocity direction. The same hypothesis is often used in exact stability codes.

These models have been inserted inside the 3-D boundary-layer code 3C3D, and allow fully automatic transition predictions in a broad range of configurations. Extension to high-lift cases with strong acceleration was realized with application to the KH3Y three elements wing. The two main advantages of these methods are that they do not require determination of initial values, and the computing time is about 2 orders of magnitude smaller compared with exact, local stability.

These methods are presented as engineering tools; they do not intend to replace exact stability codes. They are most useful in design and when parametric variations are considered, like for optimization purposes.

Emphasis has been placed here on 3-D transonic configurations, but there exists an extension of the TS model to supersonic flows up to Mach 4 . This extension was used for the highly swept JAXA wing presented in the paper. CIRA and ONERA also used the database approach in the design of the Mach 2 SUPERTRAC wing, in which case it was introduced into an optimization process.

In the case of a boundary layer computation time is very small, and in $3 \mathrm{C} 3 \mathrm{D}$ the three models and $N$-factor curves are in fact computed, allowing comparison of the results. For the integration into a RANS code, selection of a single method would be necessary to reduce computing time to a minimum because of the iterative process. Two methods are most cost effective, the envelope method and the $N_{\mathrm{TS}} / N_{\mathrm{CF} 0}$. Introduction into a RANS code has not yet been realized, although conditions for using velocity profiles extracted from RANS fields have been established.

ONERA database methods were developed for transonic aeronautic applications with emphasis on crossflow instabilities, and are not applicable to separated boundary layers. It should also be noted that the traveling crossflow model has been inserted into the ONERA local stability code CASTET to provide automatic initialization for crossflow-dominated cases, from low-speed to transonic flows, with good success. The models presented can also be used to estimate a priori the range of variation of various parameters before conducting a complete stability analysis with a local or nonlocal code.

\section{Acknowledgments}

The work presented here received support from the European Commission, under the contracts ELFIN II, EUROTRANS, EUROLIFT, and EUROLIFT II. Support was also granted from Centre National d'Etudes Spatiales and from Service des Programmes Aéronautiques. The preparation of the present paper was supported by internal ONERA funding.

\section{References}

[1] Smith, A. M. O., and Gamberoni, N., "Transition, Pressure Gradient and Stability Theory," Douglas Aircraft Co., CA Rept. ES 26388, Long Beach, CA, Sept. 1956.

[2] van Ingen, J. L., "A Suggested Semi-Empirical Method for the Calculation of the Boundary-Layer Transition Region," Univ. of Delft, Rept. VTH-74, Dept. of Aerospace Engineering, Oct. 1956.

[3] Arnal, D., "Transition Prediction in Transonic Flow," IUTAM Symposium Transsonicum III DFVLR-AVA, Springer-Verlag, Berlin, 1988, pp. 253-262.

[4] Vialle, F., "Transition Sur un Ellipsoïde de Révolution," ONERA, Student Training Rept., Sept. 1984.

[5] Stock, H.-W., and Degenhart, E., "A Simplified $e^{N}$ Method for Transition Prediction in Two-Dimensional Incompressible Boundary Layers,'Zeitschrift fur Flugwissenschaften und Weltraumforschung, Vol. 13, No. 1, 1989, pp. 16-30.

[6] Gaster, M., and Jiang, F., "Rapid Scheme for Estimating Transition on Wings by Linear Stability Theory," 19th Congress of International Council for the Aeronautical Sciences (ICAS), ICAS Paper 1994-243.

[7] Casalis, G., and Arnal, D., "ELFIN II Subtask 2.3: Database method, Development and Validation of the Simplified Method for Pure Crossflow Instability at Low Speed," ELFIN II, Technical Rept., No. 145; also ONERA, Rept. No. 119/5618.16 DERAT, Dec. 1996.

[8] Fuller, R., Saunders, W. R., and Vandsburger, U., "Neural Network Estimation of Disturbance Growth Using a Linear Stability Numerical Model," AIAA Paper 1997-0559, Jan. 1997.

[9] Crouch, J. D., Crouch, I. W. M., and Ng, L. L., "Estimating the Laminar/ Turbulent Transition Location in Three-Dimensional Boundary Layers for CFD Applications," AIAA Paper 2001-2989, Jan. 2001.

[10] Perraud, J., Donelli, R. S., and Casalis, G., "Database Approach: Treatment of Stationary Modes," ALTTA, Technical Rept. No. 24; also ONERA, Rept. No. RT 140/03828 DAAP/DMAE.

[11] Langlois, M., Masson, C., Kafyeke, F., and Paraschivoiu, I., "Automated Method for Transition Prediction on Wings in Transonic Flows," Journal of Aircraft, Vol. 39, No. 3, 2002, pp. 460-468. doi: $10.2514 / 2.2951$

[12] Drela, M., "Implicit Implementation of the Full $e^{N}$ Transition Criterion," AIAA Paper 2003-4066, Jan. 2003.

[13] Cliquet, J., Houdeville, R., and Arnal, D., "Application of LaminarTurbulent Transition Criteria in Navier-Stokes Computations," AIAA Journal, Vol. 46, No. 5, 2008, pp. 1182-1190. doi: $10.2514 / 1.30215$

[14] Perraud, J., Cliquet, J., Houdeville, R., Arnal, D., and Moens, F. "Transport Aircraft 3-D High-Lift Wing Numerical Transition Prediction," Journal of Aircraft, Vol. 45, No. 5, 2008, pp 1554-1563. doi: $10.2514 / 1.32529$

[15] Krumbein, A. M., "Automatic Transition Prediction and Application to Three-Dimensional Wing Configurations," Journal of Aircraft, Vol. 44, No. 1, 2007, pp. 119-133. doi: $10.2514 / 1.22254$

[16] Toulorge, T., Ponsin, J., Perraud, J., and Moens, F., "Automatic Transition Prediction for RANS Computations Applied to a Generic High-Lift Wing," AIAA Paper 2007-1086, Jan. 2007.

[17] Moens, F., Perraud, J., Iannelli, P., Toulorge, T., Eliasson, P., and Krumbein, A., "Transition Prediction and Impact on 3-D High-Lift Wing Configuration," Journal of Aircraft, Vol. 45, No. 5, 2008, pp. 1751-1766. doi: $10.2514 / 1.36238$

[18] Mack, L. M., "Boundary-Layer Linear Stability Theory," Special Course on Stability and Transition of Laminar Flow, AGARD Rept. No. 709, 1984.

[19] Schrauf, G., Perraud, J., Vitiello, D., and Lam, F., "Comparison of 
Boundary-Layer Transition Predictions using Flight Test Data," Journal of Aircraft, Vol. 35, No. 6, 1998, pp. 891-897. doi: $10.2514 / 2.2409$

[20] Arnal, D., Casalis, G., and Houdeville, R., "Practical Transition Prediction Methods: Subsonic and Transonic Flows," Advances in Laminar Turbulent Transition Modeling, von Karman Inst. Lecture Series, 2008 (to be published); also ONERA Rept. RF 1/13639 DMAE, Sept. 2008.

[21] Delbez, J., and Hallouard, J. M., "Etude de la Stabilité des Profils de Couche Limite Laminaire Avec Courant de Retour," ONERA, Student Training Rept., June 1986.

[22] Gregory, N., Stuart, J. T., and Walker, W. S., "On the Stability of ThreeDimensional Boundary Layer with Application to the Flow Due to a Rotating Disc," Philosophical Transactions of the Royal Society of London, Series A: Mathematical and Physical Sciences, Vol. 248, No. 943, 1955, pp. 155-199. doi:10.1098/rsta.1955.0013
[23] Gaster, M., "A Note on the Relation Between Temporally Increasing and Spatially Increasing Disturbances in Hydrodynamic Stability," Journal of Fluid Mechanics, Vol. 14, No. 2, 1962, pp. 222-224. doi:10.1017/S0022112062001184

[24] Schrauf, G., "Large-Scale Laminar Flow Tests Evaluated With Linear Stability Theory," AIAA Paper 2001-2444, Jan. 2001.

[25] Houdeville, R., Mazin, C., and Corjon, A., "Method of Characteristics for Computing Three-Dimensional Boundary Layers," La Recherche Aerospatiale, Vol. 1, 1993, pp. 37-49.

[26] Tokugawa, N., Kwak, D.-Y., Yoshida, K., and Ueda, Y., "Transition Measurements of Natural Laminar Flow Wing on Supersonic Experimental Airplane NEXT-1," Journal of Aircraft, Vol. 45, No. 5, 2008, pp. 1495-1504.

doi: $10.2514 / 1.33596$
A. Tumin

Associate Editor 